\title{
On Demand Temporary Parallel Route Recovery for Frequent Link Failure in Adhoc Networks
}

\author{
Amit D. Kothari. \\ Marwadi Education Foundation \\ Group of Institutions, \\ Faculty of Computer Applications (MCA) \\ Rajkot, India.
}

\author{
Dr. Ashok R. Patel. \\ Hemchandracharya North Gujarat University. \\ M.Sc. (IT/CA) Dept, Patan.
}

\begin{abstract}
Adhoc network is collection of wireless mobile nodes dynamically forming a temporary network without the use of existing network infrastructure of centralized administration. Each node acts as a router by itself and forwards all the packets which it receives.

This concept is basically useful when there is a very high link failure due to migration of nodes from the available network region. There is a frequent link failure in adhoc networks, which causes packet to be lost or packets doubts to reach destination. In this scenario a different mechanism and scheme is proposed and implemented to make the important time critical data like real time or voice data to reach the destination without any loss. The mechanism used is a special propagation which propagates a unique kind of parallel route discovery for real time application scenario to send the time critical data safely. The scheme used is temporary parallel route recovery builds a temporary parallel path between the nodes during link failure. The important node then forwards the buffered packets to the destination without any loss which is on-demand based on type of information a node forwards. Special buffer is allocated and simulated by using a simulation tool NS-2. This extensive mode is compared with standard models performance metrics such as packet delivery ration, routing overheads and average delay are analyzed.
\end{abstract}

\section{Keywords}

MANETS, time critical data, special propagation mechanism, temporary route repair, route maintenance [9], NS-2 [8].

\section{INTRODUCTION}

The wireless adhoc network [1] had relative congestion and flooding of packets leading to packet buffering which will continuously degrades the performance. A significant amount of information is lost due to buffering of some packets with important data. Thus it is important to explore a new algorithm which is differ from DSR [5] and AODV [2] [3] [4], to minimize the routing delay in case of intermediate node failure while traversing the packets towards the destination. The main focus is to enhance the existing route recovery concept and there by providing an efficient routing method to avoid congestion during link failures.

The first section describes the problems in adhoc networks. In second section a special propagation mechanism for significant data is explained. The next section of the paper deals with the temporary parallel route recovery mechanism. Finally the performance metrics are compared graphically.

\section{PROBLEMS IN MANETS}

Link failure is high in wireless Adhoc networks because mobility of nodes is high and nodes move dynamically in the network [6]. This degrades the performance of MANETS (Mobile Adhoc NETworks [7]) and leads to problem in transportation layer.

Problems encountered in transport layer are loss of packets and route failure. Congestion in network occurs when excess amount of packets are lost. When there is a loss of critical data like real-time data or video data which carries very sensitive information the data must be retransmitted again from the source. Retransmitting the data again from the same source will consume more bandwidth and extra power in wireless adhoc networks. When a critical data is to be transmitted to the destination and if congestion occurs in the network there will be delay for transmitting the significant data. Due to the frequent link failures in MANETs there is no guarantee that the critical data reaches the destination safely.

In the adhoc networks previous works are based on buffer allocation, buffer optimization for all the data and this paper gives importance for the time critical data which are significant in nature. The paper gives solution to the problems caused by frequent loss of time critical data in MANETs. The solutions proposed are aimed at improving the reliability in adhoc networks, consequently the rate at which data is transmitted (throughput), and minimize the delay caused by congestion in the network by buffering the critical data which are significant in nature.

\subsection{Need for the algorithm}

Generally in wireless adhoc network each and every node act as a router and forward the packet to destination. There is a possibility that the packets can be dropped by the nodes which acts as a router due to some reasons. If the time critical data is lost it cannot be recovered back from the intermediate node. Only upon the request the data can sent again from the source node. This causes delay in transmitting the data and congestion in the network. Even by selecting a single route for transmission intermediate node face a problem of route failure frequently due to the random movement of neighbor node. Through (adhoc on demand temporary parallel route recovery) AODTPRR takes the best parallel route for the destination so delay is minimized in case of route failure. 


\subsection{Assumptions}

(a) When a node is within the rediorange of other they are termed as neighbours.

(b) Each node which is holds a special buffer to store the packets dropped at the intermediate nodes.

\subsection{Proposed solutions}

In order to prevent loss of important and time critical data a mechanism along with a scheme is introduced in this paper.

\subsubsection{Special propagation mechanism}

This mechanism broadcasts a Special kind of propagation for critical data that is assumed for transmitting significant information.

\subsubsection{Temporary parallel route recovery scheme}

This scheme proposes a new temporary parallel route recovery concept for buffering the transmitting data which are dropped by intermediate nodes.

Delay caused by the link failure is minimized using the buffers allocated for the intermediate node these two schemes.

Basically there are two problems in case of link failure

\subsubsection{Packet loss}

Each and every node carrying critical data is assigned a buffer that holds the data that is dropped. Packets that are lost are buffered in the intermediate node so that when it gets the alternate route to the destination it passes the data to the destination.

\subsubsection{Packets do not reach the destination}

When a node move from one network to other network the packets forwarded to that node in that particular network are dropped and becomes unreachable.

During this situation the intermediate node that detected frequent link failure for the desire destination node will temporarily create an efficient parallel route and send the data to the destination. This helps to send the data to the destination without any delay and instant parallel route establishment takes place in this case. New temporarily efficient parallel routes are created so when after establishment of newly temporary route the sender has now two alternatives at first. Sender prefers to send the data on both the alternatives .But if any one of parallel newly established route again goes frequently failure, new temporarily parallel routes establishment procedure is performed by the intermediate node from where the connection goes failure again.

In adhoc networks though each node acts as a router and forwards the packet. The packets are not buffered and finally the information is lost. The temporary parallel route recovery scheme is a modified mechanism of local route repair in which during a link failure temporary parallel route is allocated for the nodes for transmitting significant data. This scheme gives solution for the problems caused by the frequent link failure in the Adhoc networks.

\section{SPECIAL PROPAGATION}

This special propagation scheme deals with how the time critical data that is sent from source to destination is distinguished from the standard normal propagation in MANETs. The Nodes are transmitting important time critical data like real time or video or voice. Each node that found frequent link failure propagates a special parallel route request to transmitting critical data.

When the route discovery is over the newly parallel route is stored in a route cache and the fresh is updated according to the mobility in the network.

\subsection{Generating and handling Special Parallel Route Request (TPRREQ)}

When a source wants to send time critical information to a destination and found frequent route failure for it, then it generates a TRREQ packet and broadcasts the packet to its netighbours. The TPRREQ uses the following fields in its packet.

$<$ Hop Count, TPRREQ ID, Destination IP Address, Destination Sequence Number, Originator IP Address, and Originator Sequence Number>

The hop count is the number of hops need the source to the node handling the TPRREQ. Thus when node receives a TPRREQ, if it is not the destination and nor does it has path to the destination it increments the hop count by 1 and rebroadcasts the packet to its neighbours. The destinations IP address of the destination and source generating the TPRREQ respectively. TPRREQ ID is a number that uniquely identifies the TPRREQ. If the TPRREQ ID in the TPRREQ packet matches the TPRREQ ID in the nodes route entry table the TPRREQ will be dropped if that node does not the final destination node. Destination sequence number is the greatest sequence number received in the past by the originator for any route towards the destination.

\subsection{Generating and Handling Special Parallel Route Reply (TPRREP)}

When the destination receives the TPRREQ packet it prepares a TPRREP packet increments its current destination sequence number by one and sends the TPRREP packet to the source through the nodes from which it receives the TPRREQ packets at first.

The source waits for the TPRREP for a fixed interval of time and then transmits the TPRREQ again and retries for a predefined number of times. If no response is received then the source declares that the destination is unreachable. If the source gets TPRREP it preferred the two most economical paths and all the data transmitted in that routes are taken to the destination. Each and every node that is assigned a buffer so that if there is any link failure the data that is on fly without reaching destination will be stored in the buffer of the intermediate nodes.

\subsection{Route Table Management}

The route table of a node maintains entries for each destination which is assumed to be important that receives 
critical data the node is interacting with or forwarding packets to.

The routing table has the following fields mainly

$<$ Destination IP address, Active neighbours, Number of hops1, Next hop1,Number of hops2, Next hop 2, Destination Sequence Number, paraflag,>

and Expiration time for the routing table entry.

Number of hops 1 is the hop count value for the Next hop1 neighbour. And Number of hops2is the hop count value for the Next hop2 neighbour.

Default value for Next hop2 and Number of hops2 is infinite and paraflag value is OFF.

When frequent link failure occurred the paraflag value is turn to ON and Temporary Parallel Route Request (TPRREQ) is generated and implementation of parallel route recover procedure is started.

The fields help the node to maintain the connectivity of the network. The expiration time associated with the route depends on the size of the adhoc network and indicated destination in the route table is to be removed. The node maintains the list of active neighbours that are next hop to the destination associated in the rout table. This is how this helps in transmitting time critical data.

\section{TEMPORARY PARALLEL ROUTE RECOVERY}

In adhoc network local route repair is the scheme that is wisely followed for repairing link failure in the intermediate nodes. When there is any link failure during a packet transmission a route error (RERR) is propagated to the source and the source retransmits the data. When a link failure occurs in the intermediate node the data that is to be send to the next hop may be stored in the buffer, which is taken care by TCP. A temporary parallel route discovery takes place which already exist for transmitting the buffered data. Our temporary parallel route recovery just helps to lay a temporary Parallel path to the destination and transmits the data that is buffered in the intermediate nodes.

Temporary parallel route recovery is explained with an example. Consider figure 1 which contains a route for transmitting data from A source to destination $\mathrm{X}$. If host $\mathrm{C}$ moves from one network to other network then there is a link failure in the network and the data are not further forwarded to the destination. At this time a route error is propagated to the source node before that a temporary parallel route repair takes place. A temporary parallel route recovery scheme is introduced at this point. In fact, it is very likely that a host nearby the C's original location, such as hosts $\mathrm{F}$ and $\mathrm{G}$ in the figure 1 , can serve as the relay nodes to page the gap.

To resolve this problem, the node B which is affected by link failure generates a temporary parallel route request to the neighboring nodes to reach the destination as seen in figure 2. A temporary parallel route request is broadcasted by the node B. A timer should be fixed by the initiator to track the time to live for the packet in the network. Shortest and optimal hop count is necessary to transmit the data faster to the destination.

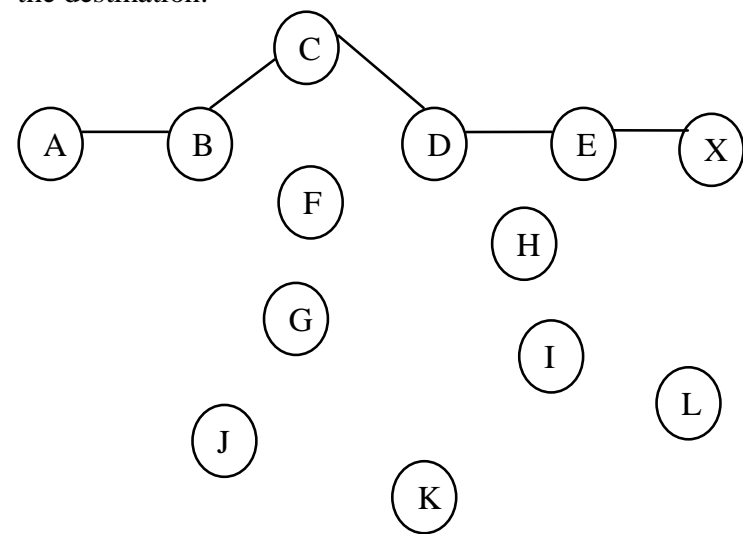

Figure 1. Node $\mathbf{C}$ moves out to other network<smiles>c1ccccc1</smiles>

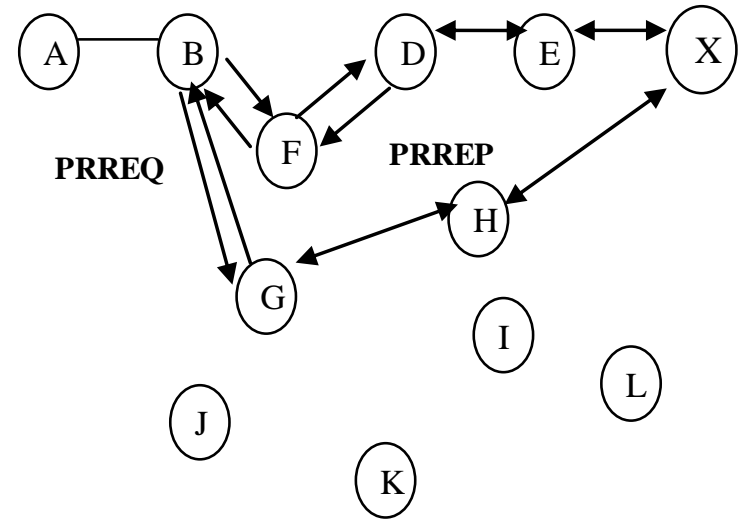

Figure 2. Temporary parallel route discovery
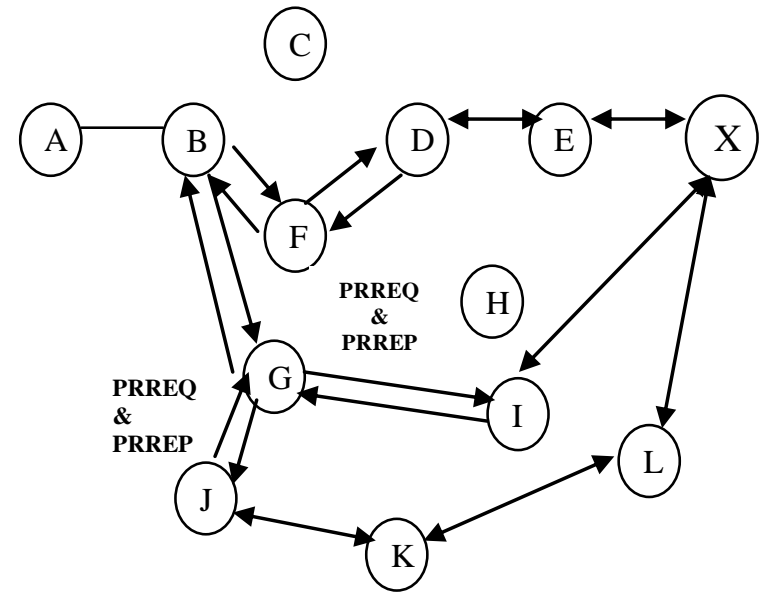

Figure 3. Temporary parallel route discovery

In figure 2 a temporary parallel route discovery for node B is shown clearly as soon as the intermediate node detects a link failure all the data that is to be transmitted are buffered and the local parallel route discovery is done. When the other 
node had receives temporary parallel route request then a temporary parallel route reply is propagated to the source from the destination. Now a temporary parallel connection is formed and the buffered data is sent safely to the destination using parallel path.

If the same problem faced by any nodes in the network i.e. $G$ in figure 3 can implement the same parallel route recover strategy to send the packet.

\subsection{Establishing path in Temporary Parallel Route Recovery}

The temporary parallel route recovery path is established and the buffered packets are sent to the destination, once a local parallel route repair occurs then the one of the temporary parallel route is replaced by a permanent route.

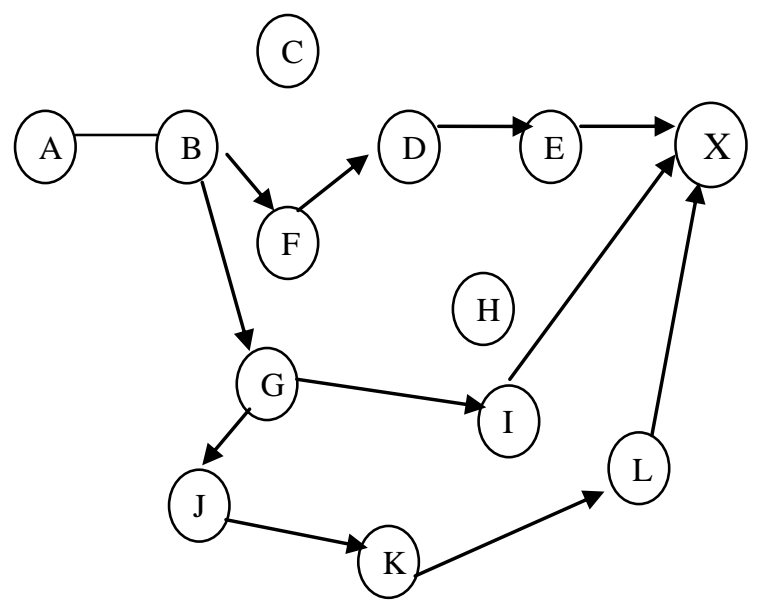

Figure 4. Temporary Parallel Route Obtained and data transmitted safely

This temporary parallel route which is shown in figure 4 shows that the buffered packets are sent to the destination safely. All the data that is buffered are sent to the destination safely. All the data that is buffered are sent to the destination and the temporary parallel route exist till the route is repaired permanently.

\section{IMPLEMENTATION}

The proposed temporary parallel route recovery was implemented using NS-2 as the simulator. The implementation part consists of following steps.

\subsection{Special propagation}

When there is any kind of link failure during the transmission the intermediate node propagates a special request and reply for the parallel route discovery. A finalized efficient parallel route is stored in a route table.

\subsection{Temporary parallel route recovery}

When there is a link failure between the intermediate nodes temporary parallel route recovery concept is introduced, a buffer is allocated for the nodes assigned and during the link failure those nodes buffer the data and the data is sent on- demand to the destination using parallel route recovery methods.

Here we can achieve speedup by transmitting the different packets on the parallel routes (i.e. Each and every packets are identified by a number and we can use the odd even selection to transfer the packet to these alternative route.) Or if the nodes are smart enough we can share the load of the node by dividing the work load in to these multiple paths, i.e. if multiple files need to be transfer we can send alternative file through these parallel route also.

\section{EXPERIMENTAL SETUP}

We preferred NS-2 version 2.33 software is for the model implementation. The simulations were based on 500 by 500 flat space scattered 5, 10, 20 and 40 wireless nodes. The nodes move from a random starting point to a destination with a speed ranging from $0-5 \mathrm{~m} / \mathrm{sec}$. The mobility model used is random waypoint in square field. Duration of simulation is 900 seconds. Simulation is done using the NS-2 simulator version 2.33 .

(1) Type - CBR.

(2)Maximum No. of connections are 25, 100, 200, 40 and 800 for the nodes 5, 10, 20 and 400 respectively.

(3) Interval between 2 CBR packets is 1 second.

(4) Simulation is implemented by considering the pause time for 5, 10, 20 and 40 nodes as 1.25, 2.50, 5, 10, 20 seconds.

(5) Maximum speed of movement is 5.0

\section{PERFORMANCE METRICS}

We used the following metrics to evaluate the performance of the temporary route recovery mechanism.

\subsection{Packet delivery ratio}

Packet delivery ratio $=$

Number of packet receives / Number of packet sends.

Packet delivery ratio is defined as the ratio of total number of packets receives and total number of packet sends.

\subsection{Routing overheads}

Routing overheads $=$ Total bytes of control message transmitted / total bytes transmitted

Routing overheads is a ratio of total bytes of control message transmitted against total bytes transmitted in the network.

\subsection{Average delay}

Average delay $=$

( $\sum$ Packet receive time $-\sum$ Packet send time) /

Total packets transmitted

\section{CONCLUSION}

When there is any link failure temporary parallel route recovery scheme is introduced and the simulation is done by using NS-2[8]. The simulation results of packet delivery ratio mentioned in table 1 and figure 5 seem equal up to number of nodes reaches to the 40 and routing overhead according to figure 6 and table 2 is not increase also though we are using parallel route. We get better result in average delay time 
according to figure 7 and table 3 in AODTPRR as compare to AODV. We can get more effective outcomes if as soon as the node which started using parallel route has increase transmission capacity.

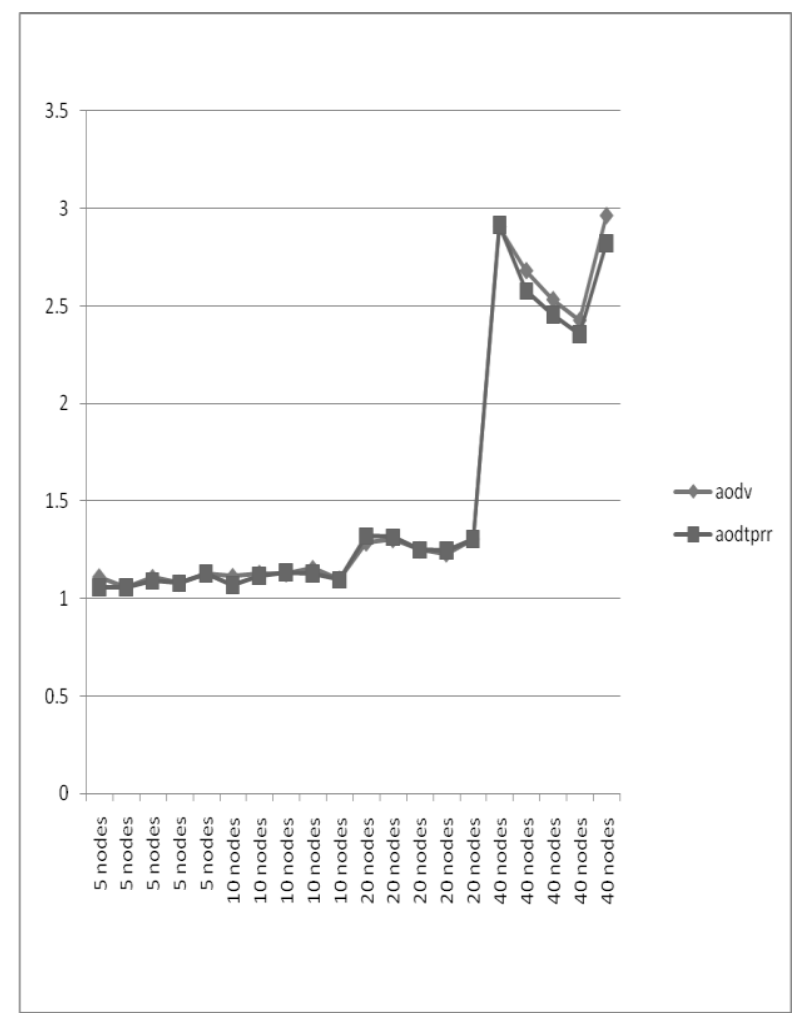

Figure 5. Comparison of packet delivery ratio of AODV and AODTPRR.

Table 1 Packet Delivery Ratio

\begin{tabular}{|l|r|r|}
\hline Nodes & \multicolumn{1}{c|}{ AODV } & \multicolumn{1}{c|}{ AODTPRR } \\
\hline 5 nodes & 1.112165162 & 1.059887784 \\
\hline 5 nodes & 1.057533064 & 1.05763060 \\
\hline 5 nodes & 1.109058286 & 1.09025148 \\
\hline 5 nodes & 1.080230693 & 1.080513544 \\
\hline 5 nodes & 1.131106981 & 1.130795886 \\
\hline 10 nodes & 1.114216585 & 1.071777769 \\
\hline 10 nodes & 1.130221304 & 1.117368763 \\
\hline 10 nodes & 1.128847911 & 1.134351638 \\
\hline 10 nodes & 1.155048252 & 1.130293226 \\
\hline 10 nodes & 1.100818333 & 1.09750341 \\
\hline 20 nodes & 1.286338512 & 1.320378107 \\
\hline 20 nodes & 1.302918191 & 1.315090043 \\
\hline 20 nodes & 1.2511911 & 1.249392139 \\
\hline 20 nodes & 1.230384565 & 1.248716605 \\
\hline 20 nodes & 1.306698322 & 1.304715344 \\
\hline
\end{tabular}

\begin{tabular}{|l|r|r|}
\hline 40 nodes & 2.904850059 & 2.91665383 \\
\hline 40 nodes & 2.681010188 & 2.577465704 \\
\hline 40 nodes & 2.533103205 & 2.455927378 \\
\hline 40 nodes & 2.426484829 & 2.3556068 \\
\hline 40 nodes & 2.962989328 & 2.821310866 \\
\hline
\end{tabular}

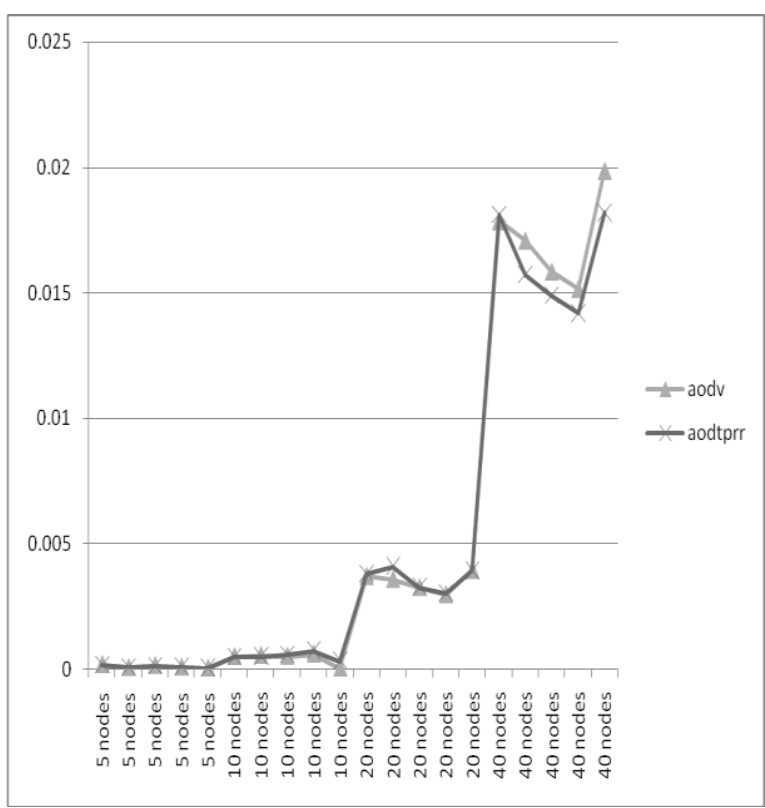

Figure 6. Comparison of routing overheads of AODV and AODTPRR

Table 2 Routing overheads

\begin{tabular}{|l|c|r|}
\hline Nodes & AODV & \multicolumn{1}{c|}{ AODTPRR } \\
\hline 5 nodes & 0.000180841 & 0.000167294 \\
\hline 5 nodes & 0.000064503 & 0.000062268 \\
\hline 5 nodes & 0.000144223 & 0.000114011 \\
\hline 5 nodes & 0.000081748 & 0.000081698 \\
\hline 10 nodes & 0.000037940 & 0.000037938 \\
\hline 10 nodes & 0.000503919 & 0.0004921 \\
\hline 10 nodes & 0.000550739 & 0.000511142 \\
\hline 10 nodes & 0.000518946 & 0.000582762 \\
\hline 10 nodes & 0.000601392 & 0.000728573 \\
\hline 20 nodes & 0.000042259 & 0.000334909 \\
\hline 20 nodes & 0.003698885 & 0.003789738 \\
\hline 20 nodes & 0.003581401 & 0.004090332 \\
\hline 20 nodes & 0.003258766 & 0.003272731 \\
\hline 20 nodes & 0.002972936 & 0.003013433 \\
\hline 40 nodes & 0.003938997 & 0.003924093 \\
\hline 40 nodes & 0.017864565 & 0.018107349 \\
\hline 40 nodes & 0.017093183 & 0.015712135 \\
\hline
\end{tabular}




\begin{tabular}{|l|r|r|}
\hline 40 nodes & 0.01585348 & 0.014882862 \\
\hline 40 nodes & 0.015148871 & 0.014175502 \\
\hline 40 nodes & 0.019881846 & 0.018192735 \\
\hline
\end{tabular}

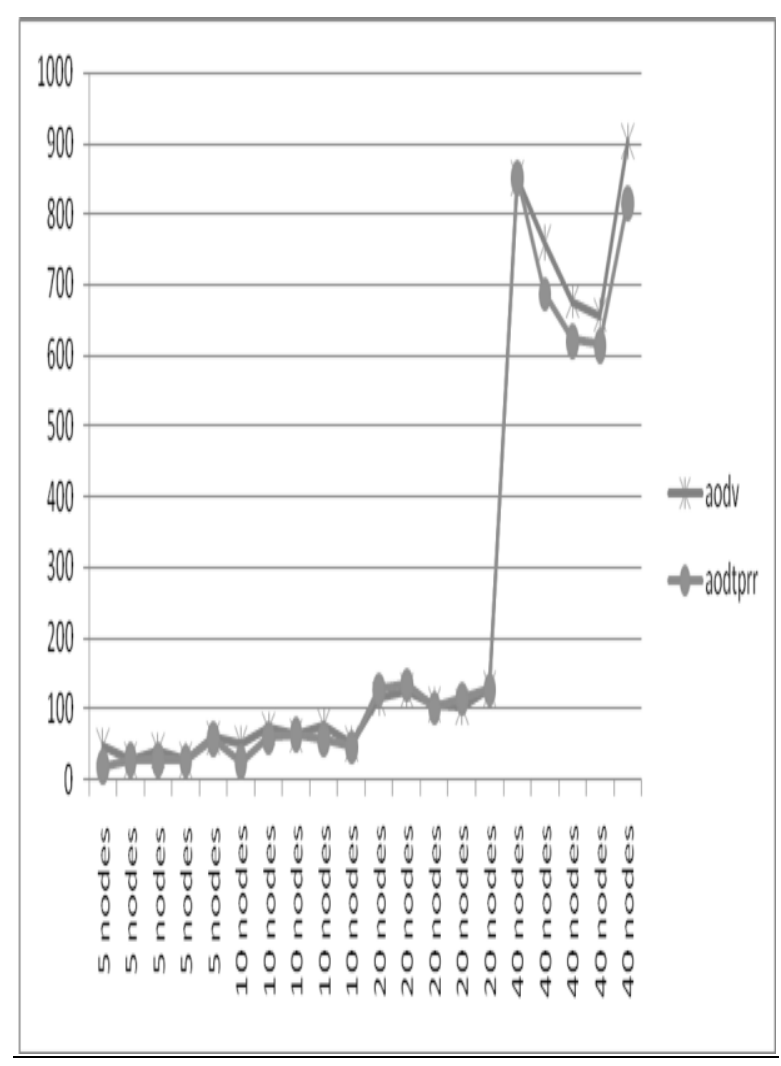

Figure 7. Comparison of Average delay of AODV and AODTPRR

Table 3 Average Delay

\begin{tabular}{|l|r|r|}
\hline Nodes & \multicolumn{1}{c|}{ AODV } & \multicolumn{1}{c|}{ AODTPRR } \\
\hline 5 nodes & 47.652134 & 18.830859 \\
\hline 5 nodes & 28.669704 & 28.669704 \\
\hline 5 nodes & 40.057317 & 30.103492 \\
\hline 5 nodes & 27.1024899 & 27.1044491 \\
\hline 5 nodes & 58.521001 & 58.520707 \\
\hline 10 nodes & 50.095328 & 26.094037 \\
\hline 10 nodes & 71.39435 & 59.546095 \\
\hline 10 nodes & 61.94572 & 64.876925 \\
\hline 10 nodes & 77.024301 & 59.233076 \\
\hline 10 nodes & 50.702005 & 47.109162 \\
\hline 20 nodes & 114.50629 & 128.453811 \\
\hline 20 nodes & 126.026998 & 135.137361 \\
\hline 20 nodes & 105.173361 & 102.672267 \\
\hline 20 nodes & 102.850689 & 115.073666 \\
\hline
\end{tabular}

\begin{tabular}{|l|r|r|}
\hline 20 nodes & 127.810053 & 128.67345 \\
\hline 40 nodes & 849.718235 & 852.903212 \\
\hline 40 nodes & 759.016212 & 688.126891 \\
\hline 40 nodes & 675.737636 & 621.13921 \\
\hline 40 nodes & 656.608678 & 614.866658 \\
\hline 40 nodes & 902.580997 & 815.770694 \\
\hline
\end{tabular}

\section{REFERENCES}

[1] Andrew S. Tanenbaum: Computer Networks, Third Edition, Prentice Hall of India Private Limited, 2000.

[2] Charles E. Perkins, Elizabeth M. BeldingRoyer, and Samir Das. "Ad Hoc On Demand Distance Vector (AODV) Routing" IETF internet draft, draft-ietfmanetaodv-10.txt, March 2002 (work in progress)

[3] C. Perkins, E. Belding-Royer, and S. Das, "Ad hoc OnDemand Distance Vector routing," RFC 3561, July 2003.

[4] C. E. Perkins and P. Bhagwat, "Highly dynamic destination-sequenced distance-vector routing (dsdv) for mobile computers," in ACM Sigcomm, London, UK, 31 August - 2 September 1994.

[5] D. Johnson, Y. Hu, and D. Maltz, "The Dynamic Source Routing Protocol (DSR) for Mobile Ad Hoc Networks for IPv4," RFC 4728, February 2007

[6] Levente Buttyan, Jeen-Pierre Hubaux "Simulating Cooperation in Adhoc Wireless Network Mobile Network and Applications" October 2003 Volume 8.

[7] L. Buttyan and J.P.Hubaux, "Stimulating Cooperation in Self-Organizing Mobile AdHOc Networks" Technical Report No.DSC/2001/046, August 2001.

[8] NS-2 manuals - http://www.isi.edu/nsnam/ns/

[9] Shih-Lin Wu, Sze-yaoNi,Yu-Chee Tseng, and Jang-Ping Sheu "Route Maintenance in a Wireless Mobile Ad Hoc Network" Proceeding of the $33^{\text {rd }}$ Hawaii International Conference on System Sciences-2000. 